\title{
ARQUEOLOGIA HISTÓRICA NA MAGNA GRÉCIA: DE UMA PERSPECTIVA AMERICANA À COLONIZAÇÃO GREGA NO SUL DA ITÁLIA*
}

Airton Pollini ${ }^{1}$

\begin{abstract}
Resumo
A abordagem da Arqueologia histórica foi forjada para o estudo da sociedade americana após a conquista europeia das terras coloniais no novo mundo. Interessada na comparação entre cultura material e registros escritos, opôs-se à Prehistória e à Antropologia e aos métodos de pesquisa dessas disciplinas. Sua principal proposta é usar todos os dados disponíveis, materiais e escritos, independentemente, sem qualquer hierarquia, mas em estreita comparação. Como tal, esta perspectiva estuda a arqueologia dos indivíduos "sem história", como indígenas, escravos, mulheres, ou mesmo as diásporas africanas na América, em antagonismo com o colonizador masculino e branco. Proposta a partir de um contexto pós-colonial explícito, esta abordagem pode dar um importante contributo para o estudo de outras sociedades antigas para as quais as interpretações se baseiam em discussões entre restos arqueológicos e textos, como as comunidades coloniais gregas na Magna Grécia.
\end{abstract}

\section{Palavras chave}

Pós-colonialismo; Arqueologia histórica; Palmares; Presidio San Francisco na Califórnia; Magna Grécia; Síbaris; Amendolara.

\footnotetext{
* Este artigo é uma versão revisada e desenvolvida da conferência proferida no séminário de doutorado Postkoloniale Antike? Alterität in den Altertumswissenschaften, Universidade de Basel (Suiça), 4 e 5 de dezembro de 2017. Agradeço as diversas questões e observações durante as discussões em Basileia, especialmente ao Prof. Dr. Tonio Hölscher e ao Prof. Dr. Christoph Ulf, aos quais sou muito grato.

1 Professor Doutor, Universidade da Haute-Alsace, Mulhouse, França. E-mail: airton.pollini@uha.fr
} 


\begin{abstract}
The historical archaeology approach was forged for the study of the American society after the European conquest of colonial lands in the new continent. Interested in the comparison between material culture and written records, it was opposed to Prehistory and Anthropology with their methods of inquiry. Its main proposal is to use all available data, material and written, independently, without any hierarchy, but in close comparison. As such, this perspective studies the archaeology of individuals "without history", such as Natives, slaves, women, or even the African diasporas in America, in antagonism to the white male European colonist. Issued from an explicit postcolonial background, this approach may give an important contribution to the study of other ancient societies where interpretations are based on discussions between archaeological remains and texts, such as the Greek colonial communities in Magna Graecia.
\end{abstract}

\title{
Keywords
}

Postcolonialism; historical archaeology; Palmares; Presidio San Francisco in California; Magna Graecia; Sybaris; Amendolara. 
As teorias pós-coloniais tornaram-se bastante populares e aceitas em várias disciplinas, depois de terem sido desenvolvidas em um campo chamado "estudos culturais". É claro que o termo e a abordagem se destinam a ser uma crítica, ou mesmo uma revolta e rebelião contra a dominação do Ocidente sobre o resto do mundo, e herdaram tanto dos movimentos de resistência colonial quanto das formas de discursos que eles geraram ${ }^{2}$.

Especificamente relacionado à colonização grega, o principal debate foi levantado após a crítica apresentada desde o famoso artigo de R. Osborne. A questão é relativamente conhecida e se concentra na colonização grega arcaica ou, como enfatizada na literatura recente de língua inglesa, a erradicação do termo "colonização" para o período arcaico. Em suma, a mobilidade grega arcaica seria uma questão de escolha individual ou de pequenos grupos que, em um primeiro momento, viveriam pacificamente em uma espécie de middle ground, onde nenhum grupo étnico seria dominante sobre os outros ${ }^{3}$. Se a bibliografia de língua inglesa geralmente adota os argumentos críticos levantados, por outro lado, esta nova visão, ou revisionismo, não foi bem recebida por outros âmbitos intelectuais, principalmente por estudiosos italianos e franceses, com uma tradição forte e já longa de estudo da colonização grega no sul da Itália, Sicília e sul da França4 ${ }^{4}$.

O principal fundamento comum da maioria dessas interpretações são, antes de tudo, as críticas a uma visão tradicional da "helenização", ou "penetrazione greca" em italiano, ou seja, a ideia da chegada dos gregos como colonos violentos com o extermínio ou a escravização de todos os indígenas. De fato, desde a década de 1960, italianos e franceses têm trabalhado para destacar vários tipos de interação e de contato entre colonos gregos e indígenas, incluindo o uso de abordagens antropológicas como "aculturação", "etnicidade", "transferências culturais", "hibridação" e outras para propor interpretações mais

\footnotetext{
2 Os principais autores que desenvolvem essa noção são: Arjun Appadurai, Homi K. Bhabha, Stuart Hall, Ania Loomba, Marshal Sahlins, Edward Said, Gayatri Spivak. Veja em particular: Bhabha, 2004; Loomba, 2005; Sahlins, 1995; Said, 1993; Said, 2003; Spivak, 1999.

3 Osborne, 1998. Diversos outro autores reforçaram essas críticas: Hall, 2012; Van Dommelen,1997; Van Dommelen , 2006; Van Dommelen, 2011; Yntema, 2000. Para uma síntese deste debate, ver Malkin, 2004.

${ }^{4}$ Veja o número especial da revista Ancient West and East de 2011, principalmente as contribuições de Domínguez, 2011 \& Greco, 2011. Veja também Greco, 2009; Greco \& Lombardo, 2010.
} 
complexas e provavelmente mais apropriadas desse processo resultante do estabelecimento de gregos além-mar ${ }^{5}$.

Em segundo lugar, e que representa muito provavelmente o principal avanço de uma perspectiva pós-colonial, há a necessidade de se identificar situações de coabitação, de um middle ground (meio termo ou terreno comum), para utilizar a expressão que Irad Malkin adaptou ao contexto grego $^{6}$. É preciso aceitar a possível existência de algumas situações em que nenhum lado é necessariamente mais poderoso do que o outro e onde uma noção como pré-colonização ou proto-colonização poderia ser operacional para a compreensão das primeiras fases de contato entre gregos e indígenas ${ }^{7}$.

No entanto, devemos estar muito vigilantes quando alguns estudiosos podem ficar tentados a estender a idéia do middle ground além dessas fases, após o período arcaico, ou em uma generalização desse processo de contatos para explicar todo o fenômeno da colonização grega no período arcaico. Esta generalização de um modo pacífico de contato ignora o fato de que, para o estabelecimento dos gregos em novos lugares, houve uma conquista real de terras, algumas das quais poderiam ter sido favorecidas ou mesmo diretamente fornecidas pela elite local. Portanto, mesmo que se reconheça a existência de alguns casos de alianças feitas por colonos e chefes locais, descritos por autores antigos mais recentes, por meio da construção de uma tradição inserida no passado, principalmente na forma de casamento misto8, não se pode apagar completamente a provável maioria de casos de violência e conquista militar. Por outro lado, se uma parte da sociedade indígena, a saber, a elite, pudesse ter

5 O congresso anual de Tarento (Taranto), no sul da Itália, funcionou como um catalizador e promotor de novas formas de interpretação da colonização grega que levam em consideração a contribuição dos indígenas na formação de uma nova sociedade colonial. Veja principalmente: Atti Taranto 1961, 1961; Atti Taranto 1967, 1967; Atti Taranto 1997, 1997, e contribuições mais recentes no caráter da colonização grega: Atti Taranto 2010, 2010; Atti Taranto 2013, 2013; Atti Taranto 2014, 2014. Veja nosso próprio comentário: Pollini, 2011. Veja também Ridgway, 2004.

${ }^{6}$ Malkin, 2002; Malkin, 2011.

7 Veja a síntese de Esposito, 2012. O debate se concentrou nas primeiras fases da colonização, com a chegada dos primeiros grupos de gregos. Veja os dois volumes das atas do congresso: Donnelan, Nizzo \& Burgers, 2016; Donnelan, Nizzo \& Burgers, 2016, e especialmente os artigos de De Angelis, 2016; Lombardo, 2016; Osborne, 2016. Em nosso próprio artigo, nós sublinhamos que a abordagem pós-colonial no âmbito intelectual de língua inglesa, em grande parte baseada em diversos filósofos franceses, foi desenvolvida quando essas teorias francesas começaram a ser criticadas, criando um certo "apartheid intelectual", cf. Esposito \& Pollini, 2016. Veja também Amselle, 2008.

${ }^{8}$ Veja a síntese de Esposito \& Zurbach, 2010. 
alianças com os colonos, principalmente para reforçar seu domínio sobre sua própria comunidade, uma parte dos indígenas de fato perderam suas terras, podem ter sido escravizados e várias mulheres devem ter sido forçadas a se casarem com colonos gregos.

Em um desenvolvimento recente do estudo da colonização grega na França, abordagens pós-coloniais resultaram na adoção, pelo menos por um curto período, de um vocabulário diferente. A transposição da palavra "diáspora" para a mobilidade dos gregos ${ }^{9}$, concebida no longo prazo, não só no período arcaico, mas até ao final do período helenístico, implicou a análise de todos os tipos de mobilidade: não apenas a instalação permanente de gregos em terras recentemente conquistadas, mas também várias outras categorias que anteriormente eram consideradas separadamente, como mercenários e soldados, artesãos, artistas, intelectuais, entre outros, estabelecidos em novas cidades gregas ou em outros contextos, como em emporia ou ao serviço de comunidades ou reinos não-gregos, na Etrúria, no Egito ou em outros lugares. No entanto, uma definição mais ampla da mobilidade grega implica a necessidade de explicar explicitamente que tipo de fenômeno está em jogo, a mobilidade individual ou de grupos, temporária ou permanente, em novos estabelecimentos gregos ou em outros contextos.

\section{Arqueologia histórica}

Além do aporte geral representado pelas perspectivas pós-coloniais, a abordagem da Arqueologia histórica pode constituir um importante contributo, destacando as várias formas de dominação e violência, mesmo em contextos de contatos culturais e de sociedades mestiças. Portanto, a Arqueologia histórica ${ }^{10}$ reintroduz os problemas das relações de poder: entre colonos e indígenas, é claro, mas também no interior de cada um desses grupos, entre elite e pobres, entre homens e mulheres ${ }^{11}$. Nesse sentido, "fluidez", "diversidade", mas também "conflito", "contradição", "dominação" e "resistência" são tópicos destacados em uma perspectiva pós-colonial e pós-moderna12.

\footnotetext{
${ }^{9} \mathrm{O}$ termo foi usado como tema para os concursos para se tornar professor das escolas públicas francessas (Agrégation and CAPES): Collin-Bouffier, 2012; Martinez-Sève, 2012; Dana, Lebreton, et al., 2012; Capdetrey \& Zurbach, 2012, veja também D'Ercole, 2012; D'Ercole, 2012.

${ }^{10}$ Funari, Hall \& Jones, 1999; Hall \& Sillman, 2006; Orser Jr, 2000; Orser Jr, 2002.

11 Gosden, 2001; Gosden, 2004; Lyons \& Papadopoulos, 2002; Voss \& Conlin Casella, 2012; Funari, Carvalho e Domínguez, 2013.

12 Rago \& Funari, 2008.
} 
Inspirada na Antropologia e nas Ciências Sociais ${ }^{13}$, e em oposição a uma abordagem antropológica da Arqueologia, essa jovem disciplina inicialmente se concentrou no estudo da sociedade americana formada após a conquista pelos europeus. Posteriormente, os campos da Arqueologia histórica se expandiram para incluir outros contextos históricos, convidando o arqueólogo a confrontar restos arqueológicos e textos ${ }^{14}$, numa abordagem que nega qualquer forma de hierarquia entre os dois tipos de dados ${ }^{15}$. Esta abordagem incentiva os estudiosos a comparar descrições escritas com a cultura material, não para encontrar o que se pode ler, mas para confrontar esses textos e tradições para propor interpretações mais nuançadas.

A este respeito, os estudos de gênero têm sido amplamente utilizados e podem ser adequadamente transpostos para a atenção dada aos restos arqueológicos deixados por qualquer grupo social tido como minoritário, no sentido político do termo, isto é, todos as frações oprimidas de uma determinada sociedade. Nesse sentido, pode ser um meio muito interessante para apreender não apenas as categorias que são definidas pelo gênero, mas, em geral, todas as categorias que se definem em oposição às camadas mais altas, à elite. É o sentido principal da abordagem das "vozes subalternas", que pretende dar substância às pessoas sem voz que deixaram suas mensagens e sua história gravadas e codificadas na cultura material. De fato, Homi K. Bhabha ${ }^{16}$ mostrou como as formas hegemônicas de controle precisam de repetição e diferenciação para serem efetivas, bem como a importância de identificar contextos de ambivalência.

\section{Quilombo de Palmares}

Em um movimento que parte da América para Magna Grécia, dois estudos de caso de contextos coloniais de interação entre colonos e outras

\footnotetext{
${ }^{13}$ Orser Jr, 2000 ; Funari, 1999.

14 Funari, 1999: 57; Funari, Zarankin \& Stovel, 2005. Veja também: Meskell, 2001; Lawrence \& Shepherd, 2006, p. 71.

15 Small, 1995: 4-5; Small, 1999: 122-136; Dyson, 1995; Johnson, 1999. No que diz respeito à Arqueologia Clássica e ao mundo grego antigo, veja: Ober, 1995; Small, 1995. 16 Bhabha, 2004, p. 29: "Not only is the social bloc heterogeneous, but, as I see it, the work of hegemony is itself the process of iteration and differentiation. It depends on the production of alternative or antagonistic images that are always produced side by side and in competition with each other. It is this side-by-side nature, this partial presence, or metonymy of antagonism, and its effective significations, that give meaning (quite literally) to a politics of struggle as the struggle of identifications and the war of positions".
} 
populações podem ajudar a distinguir alguns elementos essenciais na análise de outras realidades na Antiguidade.

O exemplo da sociedade quilômbola de Palmares é um dos pontos de ancoragem tradicional para a identidade dos antigos escravos negros e para a consciência e afirmação do empoderamento negro no Brasil atual ${ }^{17}$. Foi um assentamento bastante importante iniciado por escravos fugitivos que prosperaram entre 1605 e 1695, tendo até dez aldeias e cobrindo uma grande área no Nordeste do Brasil.

A Arqueologia histórica provou ser muito útil para a construção de uma interpretação muito mais matizada e complexa da sociedade quilômbola, ou seja, a comunidade de ex-escravos libertos, composta por várias categorias, além de negros africanos libertos e incluindo diversos tipos de pessoas, como indígenas e brancos pobres de origem europeia. Por outro lado, se os quilômbolas puderam recuperar a liberdade da escravidão imposta pelos colonos, a sociedade estava muito longe de qualquer idealização: Palmares era governada por um rei, na reprodução de alguns dos modos africanos tradicionais de organização, incluindo a exploração de indivíduos de classe baixa. A cultura material combinada com a reavaliação das fontes escritas mostrou uma imagem muito mais estratificada do que uma ideia simplista e idealista de ex-escravos negros que recuperaram a liberdade.

\section{Presídio San Francisco na Califórnia}

Tomando outro exemplo e seguindo Barbara Voss ${ }^{18}$, podemos reforçar a natureza multidimensional dos contatos entre colonos e outras populações na Califórnia. Em primeiro lugar, é necessário observar a diversidade dos povos não indígenas: neste segundo exemplo, do "Presidio San Francisco" na Califórnia, além dos colonos de origem européia, B. Voss ressalta a presença de africanos livres (não-escravos) e americanos não-indígenas provenientes de outras partes da América.

O foco da arqueologia dos contatos na distinção binária colonizadorescolonizados escondeu as diferenças sociais dentro de cada um desses grupos. Em alguns contextos, etnia ou etnicidade, gênero, religião, classe, status ou faixa etária podem ter desempenhado um papel predominante em relação à dicotomia colonizador-colonizado. Além disso, os termos utilizados para a identificação social transmitem a diferenciação. Na

\footnotetext{
17 Funari \& Carvalho, 2005.

18 Voss, 2015.
} 
Califórnia espanhola, por exemplo, o termo "soldado" contém indicação não só da ocupação, mas também de gênero, idade, nacionalidade, status colonial, hierarquia social, classe, perspectiva de capacidade econômica e física futura, em formas que não podem ser separadas umas das outras.

É muito interessante notar o funcionamento de um sistema de castas, mas esse sistema é realmente mais poroso do que se poderia pensar. No caso do "Presidio" na Califórnia, o sistema de castas permite a existência de um grande número de categorias, de acordo com o tipo de mistura étnica ou racial. Ele cria uma hierarquia complexa, em uma espécie de "pigmentocracia", na qual a cor da pele mais clara significa uma classificação mais alta na hierarquia. Este sistema é particularmente eficaz em um contexto de fronteira da dominação espanhola, onde quase nenhum colonizador tinha nascido na Europa ou tinha mesmo o pai nascido na Europa. A casta de "espanhol", já inferior à de "peninsular" (nascido na Península Ibérica), ou "criollo" (filho de europeu), dava acesso a quase todos os privilégios reservados às castas superiores e permitia uma estrutura de ascensão social a pessoas de ascendência indígena ou africana através da miscigenação. Além disso, o sistema também permitia a mudança de castas, até mesmo por intermédio de uma simples declaração.

Esta porosidade e diversidade do sistema de castas se transformou e foi então substituída pela "etnogênese" de uma nova identidade, o "califórnio". Aqui, o reconhecimento da sociedade mista não elude essas questões de violência ou dominação e a criação de uma nova identidade, a etnogênese, é concebida numa situação em que vários tipos de relações de poder ocorreram ao mesmo tempo.

No Presidio de San Francisco, os colonos eram eles próprios o produto da colonização. Eles eram os descendentes de índios mesoamericanos e africanos que tinham sido deslocados de suas terras pela colonização e que posteriormente foram mobilizados como uma força colonizadora em nome da coroa espanhola. Embora os estudos de contato tenham esmagadoramente enfatizado a pesquisa sobre o contato dos europeus com os indígenas, a situação no Presidio de San Francisco não era, de fato, atípica.

A arqueologia mostrou, no entanto, que durante as primeiras décadas da colonização, os colonos militares do Presidio de San Francisco realizaram estratégias materiais que minimizaram a representação de diferenças culturais dentro da população, ampliando a impressão de uma grande presença de europeus brancos. As comparações entre fontes textuais e 
cultura material puderam mostrar todas as nuanças e a diversidade do estabelecimento colonial.

\section{Síbaris na Magna Grécia}

Uma vez que o principal contributo teórico da Arqueologia histórica foi estabelecido, o exemplo do território de Síbaris na Magna Grécia (Fig. 1) pode esclarecer as possibilidades oferecidas por essa abordagem aplicada aos contextos coloniais antigos.

A evidência literária sobre Síbaris determina em grande parte a interpretação e, no que diz respeito ao território da cidade, a descrição de Estrabão é a mais relevante. O geográfo descreve a cidade de Síbaris, fundada por aqueus entre os rios Cratis e Síbaris e que, em seus tempos de glória [euthychia], comandava quatro povos [ethnè], tinha subjugado vinte e cinco cidades [poleis] e possuía uma muralha de cinquenta estádios. Durante o conflito com a cidade vizinha de Crotone, Síbaris contou com trezendo mil homens. Entretanto, em razão de sua soberba [tryphè] e insolência [hybris], foram privados de sua felicidade [eudaimonia] pelos crotoniatas que, em setenta dias, tomaram a cidade, desviaram o rio e a submergeram ${ }^{19}$.

No mesmo sentido, o testemunho de Diodoro da Sicília acrescenta informações sobre a concessão relativamente fácil de cidadania sibarita, quando afirma que Síbaris era a primeira entre as cidades da Itália e possuía trezentos mil cidadãos ${ }^{20}$.

A pesquisa atual é, obviamente, incapaz de determinar os quatro povos e as vinte e cinco cidades do texto de Estrabão e os trezentos mil cidadãos constituem um número muito exagerado. Por outro lado, a partir da comparação entre tradição literária e vestígios materiais, alguns pesquisadores usam a expressão "império de Síbaris" para falar sobre o

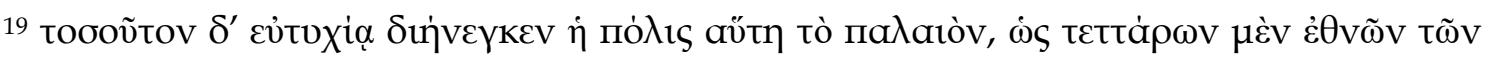

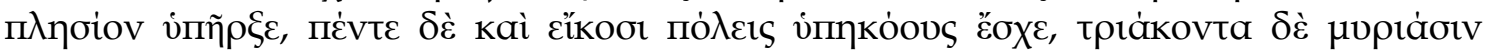

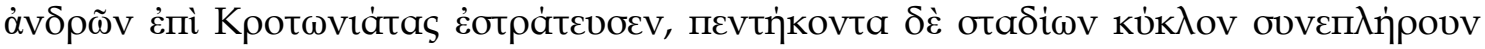

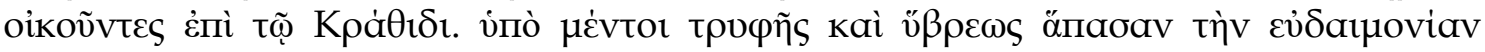

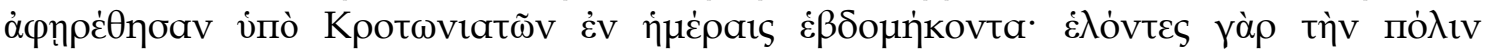

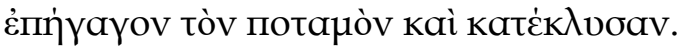

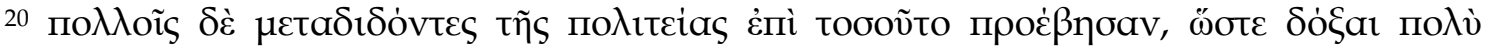

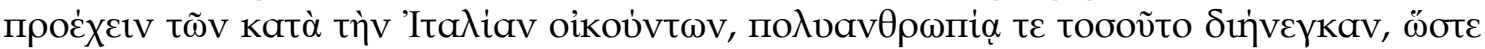

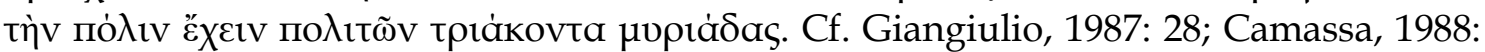
639-641; Greco, 2013: 199.
} 
seu território e suas relações com os indígenas da região ${ }^{21}$ M. Bugno ${ }^{22}$ enfatizou que o modelo desse "império de Síbaris" deve ser interpretado como comparável ao sistema hegemônico do Império Persa, onde as comunidades dominadas conservavam uma grande autonomia.

O sítio arqueológico mais importante do território de Síbaris é Francavilla Marittima, mas este é objeto de uma grande discussão, tanto no que diz respeito à área do santuário quanto da necrópole ${ }^{23}$.

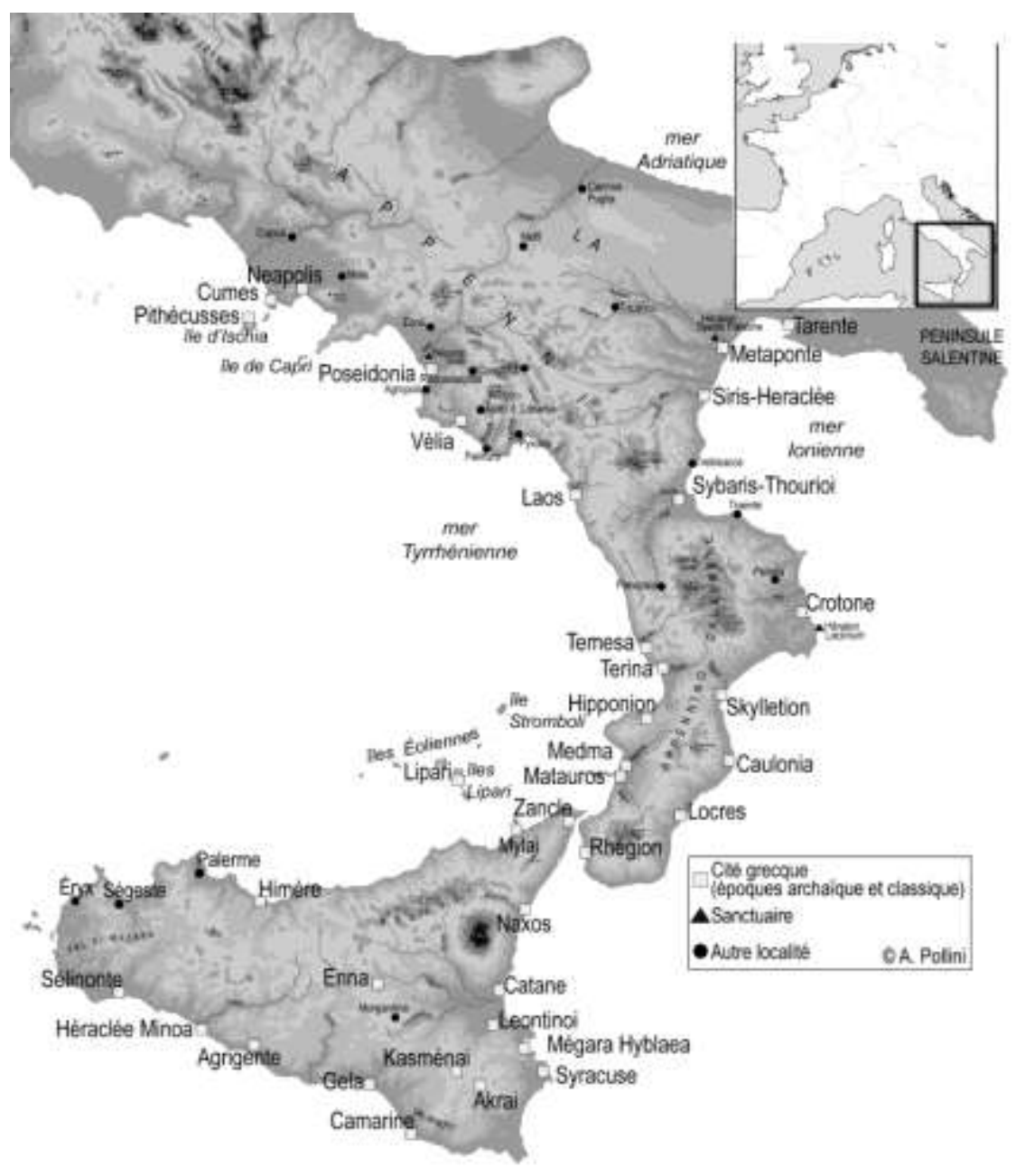

Fig. 1. Mapa da Magna Grécia e Sicília, com a indicação das cidades coloniais gregas. (C) Airton Pollini.

${ }^{21}$ Greco, 1992. Veja um exegese da tradição literária e um balanço historiográfico por Aversa, 2008. Mais recentemente, ver Greco, 2013.

22 Bugno, 2001.

${ }^{23}$ Attema, 2008; Brocato, 2012; Brocato, 2014; Granese, 2006; Granese, 2013; Guggisberg, 2014; Guggisberg, 2016; Guggisberg, Colombi \& Spichtig, 2012; Kleibrink, Jacobsen \& Handberg, 2004; Kleibrink \& Weistra, 2013; La Genière de, 1989; Luppino, Quondam, et al., 2010; Maaskant-Kleibrink, 2005; Maaskant-Kleibrink, 2006; Paoletti, 2014; Quondam, 2009. 


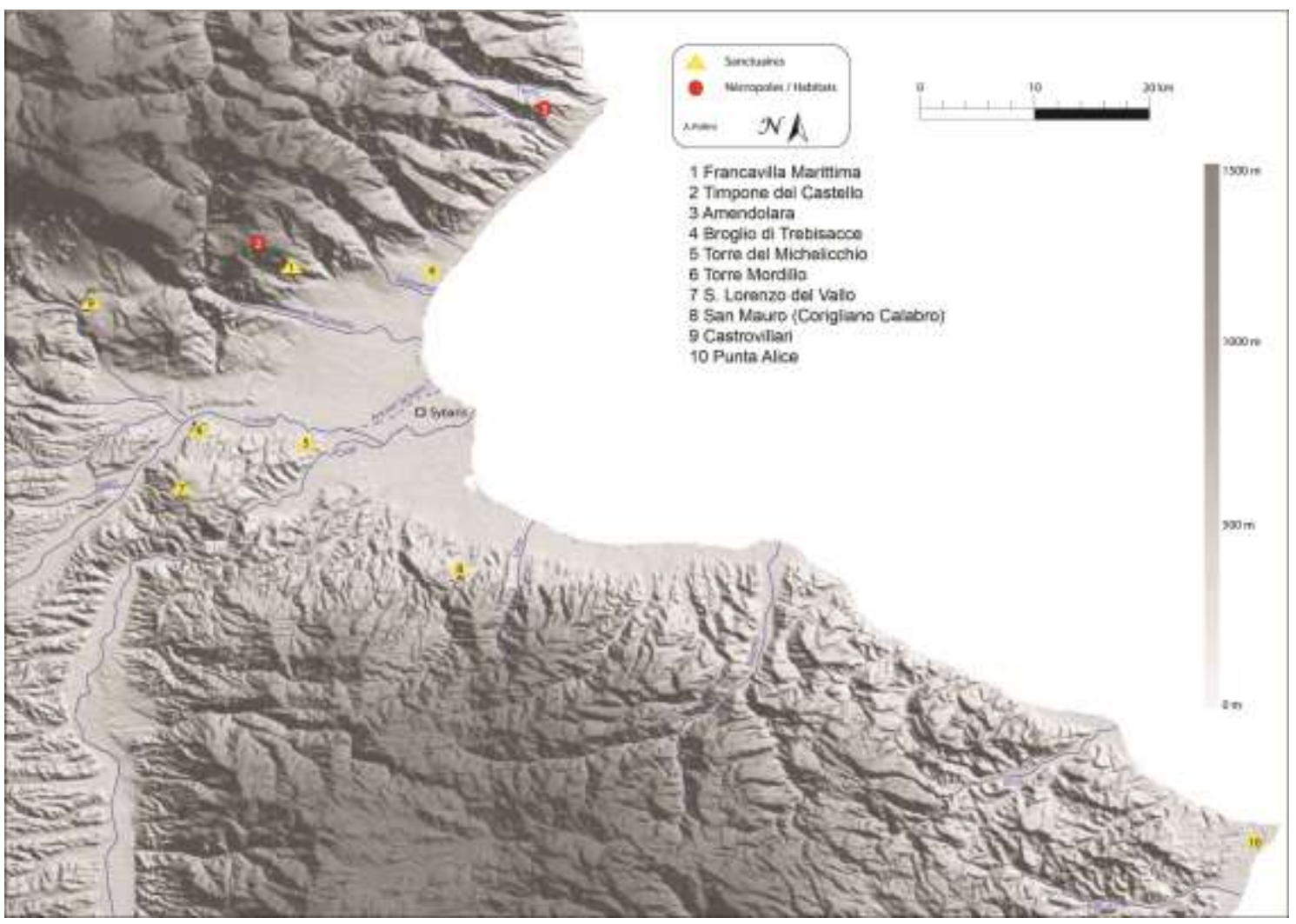

Fig. 2. Mapa do território de Síbaris com a indicação dos sítios arqueológicos: Amendolara é o número 3. (c) Airton Pollini.

Amendolara (n. 3) ${ }^{24}$ é o segundo sítio arqueológico mais importante do território de Síbaris, especialmente para a análise das relações entre gregos e indígenas. Aqui, os dados arqueológicos mostram uma realidade divergente daquela de Francavilla, o que revela a complexidade das situações e a impossibilidade de definir um modelo único para a interpretação dessas relações interétnicas em um contexto colonial.

Localizado a quase $27 \mathrm{~km}$ do centro urbano de Síbaris, Amendolara é composta de dois sítios com pelo menos dois núcleos cada. Em primeiro lugar, uma necrópole pode ser identificada na localidade Agliastroso e uma área de habitat em Rione Vecchio. Estes dois núcleos apresentam vestígios de ocupação indígena do início da Idade do Ferro, em um intervalo cronológico que vai do século IX ao final do século VIII a.C., com uma clara interrupção no final do século VIII, no momento da fundação da colônia de Síbars, quando a necrópole de Agliastroso foi

${ }^{24}$ La Genière de, 1984; La Genière de, 2012. A identificação dos vestígios arqueológicos na cidade de Amendolara data dos anos 1930, durante trabalhos agrícolas na região de Agliastroso, cf. Notizie degli Scavi, 1931, p. 654-655 and Notizie degli Scavi, 1939, p. 368369, citados por La Genière de, 2012: 9. Para uma breve síntese, ver Osanna, 1992: 163164, n. 27. 
abandonada. Apenas alguns fragmentos datáveis no século VII indicam uma reduzida continuidade do uso da área de habitat em Rione Vecchio após o final do século VIII.

O segundo sítio de Amendolara, localizado a menos de $2 \mathrm{~km}$ de distância do primeiro, é composto por duas áreas de necrópole, em Mangosa e Uomo Morto (Paladino), bem como um habitat na colina de San Nicola. A necrópole foi artificialmente dividida em duas pela construção de uma casa moderna, e apenas a porção de Paladino Oeste foi sistematicamente escavada entre 1967 e 1975, e apresenta um total de 329 túmulos totalmente publicados ${ }^{25}$. A cronologia do Paladino Oeste começa na Idade do Ferro e algumas das mais recentes tumbas se sobrepõem às mais antigas, o que permitiu algumas hipóteses de enclaves familiares, embora a ausência de muretos de delimitação não permita afirmá-las com precisão ${ }^{26}$.

O ritual fúnebre mostra pouca variação durante o uso e a forma mais comum é a da pseudo-cista: pequenas paredes laterais compostas de lajes irregulares que cercam a vala funerária, com uma laje no final onde a cabeça descansava, na maioria das vezes no oeste. É óbvio que uma necrópole usada por dois séculos também apresenta inúmeras anomalias, analisadas em detalhes por J. de La Genière27.

O ritual funerário das tumbas datadas até o início do século VII é atribuído ao contexto indígena e parece reservado à elite local. A partir da segunda metade do século VII, objetos de metal, armas em túmulos masculinos e ornamentos em tumbas femininas, desapareceram gradualmente e, no final do século, o mobiliário funerário era composto quase exclusivamente de cerâmica. O sítio é então interpretado como um grupo indígena aliado ou em uma relação de dependência com os colonos gregos.

$\mathrm{Na}$ colina de S. Nicola, a pesquisa arqueológica identificou dois terraços ocupados por casas datadas entre o primeiro terço do século VI e a última década do mesmo século, no momento da destruição de Síbaris por Crotone, em 511 a.C. No terraço superior, as casas são grandes e seguem uma orientação determinada por caminhos ou ruas retas. As técnicas de construção são comparáveis às casas arcaicas do centro urbano de Síbaris, encontradas no sítio de Stombi28. As casas no terraço inferior não são tão regulares em sua orientação, talvez por causa da maior inclinação da

${ }^{25}$ La Genière de, 2012.

26 La Genière de, 2012: 235.

27 La Genière de, 2012: 237-241.

${ }^{28}$ Cf. Sibari $V, 1992$. 
encosta nessa parte. As características do habitat de S. Nicola parecem corroborar a hipótese de um estabelecimento indígena cujas relações com os colonos gregos mostram um contexto de aliança ou de dependência.

A chegada dos colonos no final do século VIII causou uma primeira destruição do centro indígena em Agliastroso e Rione Vecchio, em um padrão semelhante aos outros sítios do território de Síbaris. Por outro lado, a particularidade de Amendolara é a persistência do elemento indígeno perto do local original e refletindo uma relação próxima com os colonos gregos. A reorganização da área após a conquista grega parece beneficiar uma parte da população nativa, como se vê no caso do habitat de S. Nicola.

Podemos, portanto, propor uma interpretação para a particularidade de Amendolara. Este estabelecimento indígeno se localizava a uma distância maior do centro urbano de Síbaris e essa distância provavelmente implicava mais dificuldade para um controle direto das terras pelos colonos gregos. O domínio em um território maior exigia alguma forma de acordo das populações nativas, pelo menos de sua elite.

Além de Francavilla e Amendolara, os outros sítios arqueológicos do território de Síbaris são relativamente mal conhecidos e, em sua maioria, são fruto de descobertas fortuitas, pesquisadas de forma limitada e não sistemática ${ }^{29}$. Eles não acrescentam muito para nosso próprio propósito e análise aqui.

\section{Conclusão}

Na nossa discussão sobre os conceitos teóricos para apreender as várias formas de interação entre colonos, indígenos e outros imigrantes, um problema complexo é determinar a proporção de cada grupo étnico e social em um determinado contexto. Em relação a Amendolara, nosso estudo de caso, quantas tumbas, quão importante era a ocupação indígena deste estabelecimento? Em geral, quais poderiam ser as estimativas do número de indígenas que vivem na área que se tornou o território da cidade de Síbaris? Um excelente contributo para a discussão consistiria em estimativas precisas e confiáveis. Elas poderão dar uma certa ideia da necessidade de violência na conquista de terras aráveis anteriormente exploradas por nativos e que se tornaram possessão grega. Tendo em conta que apenas uma parte da necrópole de Paladino (Oeste) restituiu um total de 329 túmulos distribuídos em dois séculos de

${ }_{29}^{2}$ Veja uma síntese em Luppino, 1996. Veja também uma análise por Greco, 1992. 
ocupação, o estabelecimento pode ser considerado bastante relevante. Ele parece ser suficiente para podermos afirmar o uso de um importante grau de violência na conquista da terra, diretamente ou em estado de dependência, como parece ser o caso em Amendolara.

$\mathrm{Na}$ ausência de dados quantitativos mais precisos, nossa intenção foi, antes de tudo, propor um quadro teórico que possa ajudar as nossas interpretações da evolução dos estabelecimentos gregos arcaicos. Portanto, a perspectiva da Arqueologia histórica pode ser transposta dos contextos modernos americanos para a Antiguidade Clássica. Nesse sentido, tentamos mostrar, em primeiro lugar, quão complexas as situações antigas podem ter sido. Em segundo lugar, e esta é a questão principal, quando se introduz uma perspectiva pós-colonial para o estudo da colonização grega, é essencial reforçar todas as relações de poder que podiam estar em jogo ao mesmo tempo. Podemos agora destacar a presença de elementos de origens indígenas e gregas, aos quais é muito importante adicionar todos os outros tipos de contribuição, como outros imigrantes que também se estabeleceram nas novas cidades gregas ou populações em diferentes formas de mobilidade que deixaram sinais importantes de suas visitas frequentes, sejam eles etruscos, egípcios, fenícios / cartagineses ou outros. Além do reconhecimento de várias formas de interação cultural, desde a pura violência e conquista até algum tipo de convivência, é essencial apreender todas as formas de relações de poder, não só entre colonos gregos e indígenas, tidos como grupos uniformes, mas sim dentro de cada grupo. A distinção não é apenas étnica, mas é principalmente entre estratos superiores e inferiores. Exemplos de sítios americanos analisados pela Arqueologia histórica apontam para várias categorias intermediárias e matizadas, e, na arqueologia da colonização grega, devemos procurar também pelas facções de gregos pobres e pelas elites indígenas ricas, bem como por todas as condições intermediárias possíveis.

\section{Referências bibliográficas}

Alle origini della Magna Grecia: mobilità, migrazioni, fondazioni. Atti del Convegno di studi sulla Magna Grecia, L, 2010, Tarente: Istituto per la Storia e l'Archeologia della Magna Grecia

AMSELLE, Jean-Loup. L'Occident décroché : enquête sur les postcolonialismes, coll. Un Ordre d'idées, Paris: Stock, 2008. 
ATTEMA, Peter. Conflict or coexistence? Remarks on indigenous settlements and Greek colonization in the foothills and hinterland of the Sibaritide (Northern Calabria, Italy), in BILDE, Pia Guldager \& PETERSEN, Jane Hjarl (eds.). Meetings of cultures in the Black sea region, Aarhus: Aarhus university press, 2008, p. 67-99.

AVERSA, Fabio. Società e politica a Sibari nel VI sec. a.C., Istituto di archeologia e storia antica (Lecce, Italie). Quaderni dell'Istituto di archeologia e storia antica. Università di Lecce, ISSN 0394-5995, 12, 2008, p. 7-47.

BHABHA, Homi K. The Location of culture, coll. Routledge classics, Londres: Routledge, 2004.

BROCATO, Paolo. Verso una rilettura critica degli scavi della necropoli enotria di Macchiabate a Francavilla Marittima (CS). Convivenze etniche, scontri e contatti di culture in Sicilia e Magna Grecia, coll. Aristonothos. Scritti per il Mediteraneo antico, 7, Trento: Tangram, 2012, p. 423-443.

BROCATO, Paolo (ed.). Studi sulla necropoli di Macchiabate a Francavilla Marittima (Cs) e sui territori limitrofi. Ricerche, Supplementi, 5, Arcavacata di Rende: Università della Calabria, 2014.

BUGNO, Maurizio. Strabone VI, 1, 13 C. 263 e l'ỏpxท่ di Sibari, in BUGNO, Maurizio \& MASSERIA, Concetta (eds.). Il mondo enotrio tra VI $e$ $V$ secolo a.C., coll. Atti dei seminari napoletani, 1996-1998, Quaderni di ostraka, 1, Naples: Loffredo, 2001, p. 303-327.

CAMASSA, Giorgio. La codificazione delle leggi e le istituzioni politique delle città greche della Calabria in età arcaica e classica, in SETTIS, Salvatore (ed.). Storia della Calabria. Vol. 1, Storia della Calabria antica, Rome: Gangemi, 1988, p. 613-656.

CAPDETREY, Laurent \& ZURBACH, Julien (eds.). Mobilités grecques. Mouvements, réseaux, contacts en Méditerranée, de l'époque archaïque à l'époque hellénistique. Scripta Antiqua, 46, Bordeaux: Ausonius, 2012.

COLLIN-BOUFFIER, Sophie (ed.). Les diasporas grecques du détroit de Gibraltar à l'Indus, VIIIe siècle av. J.-C.-fin du IIIe siècle av. J.-C. Pour les concours. Série Cours, Paris: Sedes, 2012.

Confini e frontiera nella grecità d'Occidente. Atti del Convegno di Studi sulla Magna Grecia, XXXVII, 1997, Tarente: Istituto per la Storia e l'Archeologia della Magna Grecia, 1999.

D'ERCOLE, Cecilia. L'essaimage colonial grec en Méditerranée antique : espaces, réseaux économiques, interactions culturelles, Annuaire. Comptes 
rendus des cours et conférences, École des Hautes Études en Sciences Sociales, 2010-2011, 2012, p. 290-291.

D'ERCOLE, Maria Cecilia. Histoires méditerranéennes : aspects de la colonisation grecque de l'Occident à la mer Noire, VIII-IVe siècles av. J.-C., coll. Collection Les Hespérides, Paris: Errance, 2012.

DANA, Madalina, LEBRETON, Stéphane, et alii. Les diasporas grecques : VIII'-III ${ }^{e}$ s., coll. Clefs concours. Histoire ancienne, Neuilly: Atlande, 2012.

DE ANGELIS, Franco. E pluribus unum: the multiplicity of models, in DONNELLAN, Lieve, NIZZO, Valentino \& BURGERS, Gert-Jan (eds.). Conceptualising early colonisation, Contextualising early colonisation II, Bruxelles: Institut historique belge de Rome, 2016, p. 97-104.

DOMÍNGUEZ, Adolfo J. The origins of Greek colonisation and the Greek polis: some observations, Ancient West and East (AWE), 10, 2011, p. 195207.

DONNELLAN, Lieve, NIZZO, Valentino \& BURGERS, Gert-Jan (eds.). Contexts of early colonization. Contextualizing early colonization, 1, Rome: Palombi, 2016.

DONNELLAN, Lieve, NIZZO, Valentino \& BURGERS, Gert-Jan (eds.). Conceptualising early colonisation. Contextualizing early colonization, 2, Bruxelles: Belgisch Historisch Insituut te Rome, 2016.

DYSON, Stephen. Is there a text in this site?, in SMALL, David B. (ed.). Methods in the Mediterranean: historical and archaeological views on texts and archaeology, Leiden: Brill, 1995, p. 25-44.

ESPOSITO, Arianna. La question des implantations grecques et de la précolonisation en Italie du Sud : entre emporia et apoikiai, in MARTINEZSÈVE, Laurianne (ed.). Les diasporas grecques du VIIIe à la fin du IIIe siècle av. J.-C., coll. Pallas, 89, Toulouse: Presses universitaires du Mirail, 2012, p. 97-121.

ESPOSITO, Arianna \& POLLINI, Airton. Post-colonialism from America to Magna Graecia, in DONNELLAN, Lieve, NIZZO, Valentino \& BURGERS, Gert-Jan (eds.). Conceptualising early colonisation, Contextualising early colonisation, II, Bruxelles: Institut historique belge de Rome, 2016, p. 61-75.

ESPOSITO, Arianna \& ZURBACH, Julien. Femmes indigènes et colons grecs : quelques observations, in ROUILLARD, Pierre (ed.). Portraits de 
migrants, portraits de colons, coll. Colloques de la Maison René-Ginouvès, 6, 2, 2010, p. $51-70$

FUNARI, Pedro Paulo Abreu. Historical archaeology from a world perspective, in FUNARI, Pedro Paulo Abreu, HALL, Martin \& JONES, Siân (eds.). Historical Archaeology: back from the edge, Londres: Routledge, 1999, p. 37-66.

FUNARI, Pedro Paulo Abreu \& CARVALHO, Aline Vieira de. Palmares, ontem e hoje, Rio de Janeiro, RJ: Zahar, 2005.

FUNARI, Pedro Paulo Abreu, CARVALHO, Aline Vieira de \& DOMÍNGUEZ, Lourdes (eds.). Arqueología de contacto: diálogos latinoamericanos, Campinas: Unicamp, 2013.

FUNARI, Pedro Paulo Abreu, HALL, Michael \& JONES, Sîan (eds.). Historical Archaeology: back from the edge. One world archaeology, 31, Londres: Routledge, 1999.

FUNARI, Pedro Paulo Abreu, ZARANKIN, Andrés \& STOVEL, Emily (eds.). Global archaeological theory: contextual voices and contemporary thoughts, New York: Kluwer academic/Plenum publishers, 2005.

GIANGIULIO, Maurizio. Aspetti di sotria della Magna Grecia arcaica e classica fino alla guerra del Peloponneso, in PUGLIESE CARRATELLI, Giovanni (ed.). Magna Grecia. 2 Lo sviluppo politico, sociale ed economico, Milan: Electa, 1987, p. 9-54.

GOSDEN, Chris. Postcolonial archaeology: issues of culture, identity, and knowledge, in HODDER, Ian (ed.). Archaeological theory today, Cambridge: Polity, 2001, p. 241-261.

GOSDEN, Chris. Archaeology and colonialism: cultural contact from 5000 BC to the present, coll. Topics in contemporary archaeology, Cambridge: Cambridge University Press, 2004.

GRANESE, Maria Tommasa. Culto e pratiche rituali nel santuario arcaico di Francavilla Marittima (Sibari - CS), Annuario della Scuola archeologica di Atene e delle missioni italiane in oriente, LXXXIV, s. III, 6, t. 1, 2006, p. 417463.

GRANESE, MARIA TOMMASA. Un luogo di culto del territorio di Sibari: il santuario di Francavilla Marittima (CS), in DELIA, Giorgio \& MASNERI, Tullio (eds.). Sibari : archeologia, storia, metafora, coll. Quaderni del liceo, 2, Castrovillari: Il coscile, 2013, p. 57-84. 
Greci e Italici in Magna Grecia. Atti del Convegno di studi sulla Magna Grecia, I, 1961, Tarente: Istituto per la Storia e l'Archeologia della Magna Grecia

GRECO, Emanuele. L'impero di Sibari: bilancio archeologico-topografico. Sibari e la Sibaritide. Atti del convegno di studi sulla Magna Grecia, XXXII, 1992, Tarente, Naples: Istituto per la Storia e l'Archeologia della Magna Grecia, 1993, p. 459-485.

GRECO, Emanuele. Le esperienze coloniali greche : modelli e revisioni: introduzione ai lavori in FRISONE, Flavia \& LOMBARDO, Mario (éds.). Colonie di colonie. Le fondazioni sub-coloniali greche tra colonizzazione e colonialismo, 2009, Lecce: Congedo editore, p. 9-16.

GRECO, Emanuele. On the Origin of the Western Greek Poleis, Ancient West and East (AWE), 10, 2011, p. 233-242.

GRECO, Emanuele. Sul cosiddetto 'impero' di Sibari fino alla tirannide di Telys ed alla distruzione della città, in DELIA, Giorgio \& MASNERI, Tullio (eds.). Sibari : archeologia, storia, metafora, coll. Quaderni del liceo, 2, Castrovillari: Il coscile, 2013, p. 197-203.

GRECO, Emanuele \& LOMBARDO, Mario. La colonizzazione greca: modelli interpretativi nel dibattito attuale. Alle origini della Magna Grecia: mobilità, migrazioni, fondazioni. Atti del Convegno di studi sulla Magna Grecia, L, 2010, Tarente, Naples: Istituto per la Storia e l'Archeologia della Magna Grecia, p. 37-60.

GUGGISBERG, Martin A. The Macchiabate necropolis in Francavilla Marittima (Calabria): some preliminary notes on the recent excavations by the university of Basel, in NIJBOER, Albert, WILLEMSEN, Sarah Lea, et alii (eds.). Macchiabate necropolis in Francavilla Marittima, coll. Caeculus, 8, Leuven: Peeters, 2014, p. 153-167.

GUGGISBERG, Martin A. Local identity and cultural exchange in (pre-) colonial Francavilla Marittima: the Macchiabate necropolis in the light of new excavations, in DONNELLAN, Lieve, NIZZO, Valentino \& BURGERS, Gert-Jan (eds.). Contexts of early colonization, coll. Acts of the conference "Contextualizing early colonization : archaeology, sources, chronology and interpretative models between Italy and the Mediterranean", vol. 1. Papers of the Royal Netherlands Institute in Rome, 64, Roma: Palombi, 2016, p. 237-246.

GUGGISBERG, Martin A., COLOMBI, Camilla \& SPICHTIG, Norbert. Gli scavi dell'Università di Basilea nella necropoli enotria di Francavilla Marittima, Bollettino d'Arte, 97, fasc. 15, 2012, p. 1-18. 
HALL, Jonathan. Early Greek settlement in the west: the limits of colonialism, in BOSHER, Kathryn (ed.). Theatre Outside Athens: Drama in Greek Sicily and South Italy, Cambridge: Cambridge University Press, 2012, p. 19-34.

HALL, Martin \& SILLIMAN, Stephen W. (eds.). Historical archaeology. Blackwell studies in global archaeology, Malden, MA: Blackwell Publishing, 2006.

Ibridazione e integrazione in Magna Grecia: forme modelli dinamiche. Atti del Convegno di studi sulla Magna Grecia, LIV, 2014, Tarente: Istituto per la storia e l'archeologia della Magna Grecia

JOHNSON, Matthew H. Rethinking historical archaeology, in FUNARI, Pedro Paulo Abreu, HALL, Martin \& JONES, Siân (eds.). Historical Archaeology: back from the edge, Londres: Routledge, 1999, p. 23-36.

KLEIBRINK, Marianne, JACOBSEN, Jan Kindberg \& HANDBERG, Søren. Water for Athena: votive gifts at Lagaria (Timpone della Motta, Francavilla Marittima, Calabria), World Archaeology, 36, n. 1, The Object of Dedication, 2004, p. 43-67.

KLEIBRINK, Marianne \& WEISTRA, Elizabeth. Una dea della rigenerazione, della fertilità e del matrimonio. Per una ricostruzione della dea precoloniale della Sibaritide, in DELIA, Giorgio \& MASNERI, Tullio (eds.). Sibari : archeologia, storia, metafora, coll. Quaderni del liceo, 2, Castrovillari: Il coscile, 2013, p. 35-55.

La città e il suo territorio. Atti del convegno di studi sulla Magna Grecia, VII, 1967, Tarente: Istituto per la Storia e l'Archeologia della Magna Grecia, 1968.

LA GENIÈRE De, Juliette. Amendolara, in NENCI, Giuseppe \& VALLET, Georges (eds.). Bibliografia topografica della colonizzazione greca in Italia e nelle isole tirreniche, III, siti Abaceno-Bari, Pisa: Scuola normale superiore, 1984, p. 210-214.

LA GENIÈRE De, Juliette. Francavilla Marittima, in NENCI, Giuseppe \& VALLET, Georges (eds.). Bibliografia topografica della colonizzazione greca in Italia e nelle isole tirreniche, VII, siti Cuccuvà-Garaguso, Pisa: Scuola normale superiore, 1989, p. 492-497.

LA GENIÈRE De, Juliette. Amendolara : la nécropole de Paladino Ouest, coll. Collection du Centre Jean Bérard, 39, Naples: Centre Jean Bérard, 2012. 
LAWRENCE, Susan \& SHEPHERD, Nick. Historical archaeology and colonialism, in HICKS, Dan \& BEAUDRY, Mary C. (eds.). The Cambridge companion to historical archaeology, Cambridge: Cambridge University Press, 2006, p. 69-86.

LOMBARDO, Mario. Le 'prime fondazioni' greche in Occidente : tradizioni antiche e letture moderne, in DONNELLAN, Lieve, NIZZO, Valentino \& BURGERS, Gert-Jan (eds.). Contexts of early colonization, coll. Acts of the conference "Contextualizing early colonization : archaeology, sources, chronology and interpretative models between Italy and the Mediterranean", vol. 1. Papers of the Royal Netherlands Institute in Rome, 64, Roma: Palombi, 2016, p. 261-273.

LOOMBA, Ania. Colonialism-postcolonialism, 2 a ed., Londres: Routledge, 2005.

LUPPINO, Silvana. I Santuari dispersi. Santuari della Magna Grecia in Calabria, coll. I Greci in Occidente, Naples: Electa, 1996, p. 221-224.

LUPPINO, Silvana, QUONDAM, Francesco, et alii. Sibaritide: riletture di alcuni contesti funerari tra VIII e VII sec. a.C. Alle origini della Magna Grecia: mobilità, migrazioni, fondazioni. Atti del Convegno di studi sulla Magna Grecia, L, 2010, Tarente, Naples: Istituto per la Storia e l'Archeologia della Magna Grecia, p. 643-682.

LYONS, Claire L. \& PAPADOPOULOS, John K. (eds.). The archaeology of colonialism, Los Angeles: Getty Research Institute, 2002.

MAASKANT-KLEIBRINK, Madeleine. The early Athenaion at Lagaria (Francavilla Marittima) near Sybaris: an overview of its early-geometric II and its mid-7th century BC phases. Papers in Italian archaeology. 6. Communities and settlements from the Neolithic to the Early Medieval Period. Proceedings of the 6th conference of Italian archaeology held at the University of Groningen, Groningen Institute of Archaeology, the Netherlands, April 15-17, 2003, BAR. International series, 1452, 2005, Oxford: Archaeopress, p. 754772 .

MAASKANT-KLEIBRINK, Madeleine. Oenotrians at Lagaria near Sybaris. A native proto-urban centralised settlement : a preliminary report on the excavation of timber dwellings on the Timpone della Motta near Francavilla Marittima (Lagaria), Southern Italy, coll. Accordia specialist studies on Italy, 11, Londres: Accordia Research Centre, University of London, 2006.

MALKIN, Irad. A colonial Middle Ground: Greek, Etruscan, and local elites in the Bay of Naples, in LYONS, Claire L. \& PAPADOPOULOS, 
John K. (eds.). The archaeology of colonialism, Los Angeles: Getty Research Institute, 2002, p. 151-181.

MALKIN, Irad. Postcolonial Concepts and Ancient Greek Colonization, MLQ: Modern Language Quarterly, vol. 65, n. 3, 2004, p. 341-364.

MALKIN, Irad. A Small Greek World: networks in the Ancient Mediterranean, Oxford: Oxford University Press, 2011.

MARTINEZ-SÈVE, Laurianne (ed.). Les Diasporas grecques du VIIIe à la fin du III e siècle av. J.-C. Pallas, 89, Toulouse: Presses universitaires du Mirail, 2012.

MESKELL, Lynn. Archaeologies of identity, in HODDER, Ian (ed.). Archaeological theory today, Cambridge: Polity, 2001, p. 187-213.

OBER, Josiah. Greek Horoi: artifactual texts and the contingency of meaning, in SMALL, David B (ed.). Methods in the Mediterranean: historical and archaeological views on texts and archaeology, Leiden: Brill, 1995, p. 91123.

ORSER Jr, Charles E. Introducción a la arqueología histórica, Trad.ZARANKIN, Andrés, Buenos Aires: Asociación amigos del Instituto nacional de antropología, 2000.

ORSER Jr, Charles E. (ed.). Encyclopaedia of Historical Archaeology, Londres: Routledge, 2002.

OSANNA, Massimo. Chorai coloniali da Taranto a Locri : documentazione archeologica e ricostruzione storica, Rome: Istituto Poligrafico e Zecca dello Stato, 1992.

OSBORNE, Robin. Early Greek Colonization? The Nature of Greek Settlement in the West, in FISHER, Nick \& VAN WEES, Hans (eds.). Archaic Greece: New Approaches and New Evidence, Londres: Duckworth, 1998, p. 251-269.

OSBORNE, Robin. Greek 'colonisation': what was, and what is, at stake?, in DONNELLAN, Lieve, NIZZO, Valentino \& BURGERS, Gert-Jan (eds.). Conceptualising early colonisation, Contextualising early colonisation II, Bruxelles: Institut historique belge de Rome, 2016, p. 21-26.

PAOLETTI, Maurizio. Presentazione. La necropoli enotria di Macchiabate, Lagaria e la 'dea di Sibari', in BROCATO, Paolo (ed.). Studi sulla necropoli di Macchiabate a Francavilla Marittima (Cs) e sui territori 
limitrofi, coll. Ricerche, Supplementi, 5, Arcavacata di Rende: Università della Calabria, 2014, p. 7-21.

Poleis e politeiai nella Magna Grecia arcaica e classica. Atti del Convegno di studi sulla Magna Grecia, LIII, 2013, Tarente: Istituto per la Storia e l'Archeologia della Magna Grecia

POLLINI, Airton. Les congrès de Tarente et les thèmes de recherche sur la Grande Grèce, Mélanges de l'École française de Rome. Antiquité, 123-2, 2011, p. $423-432$.

QUONDAM, Francesco. La necropoli di Francavilla Marittima: tra mondo indigeno e colonizzazione greca, in BETTELLI, Marco, De FAVERI, Cecilia \& OSANNA, Massimo (eds.). Prima delle colonie : organizzazione territoriale e produzioni ceramiche specializzate in Basilicata e in Calabria settentrionale ionica nella prima età del ferro, coll. Atti delle giornate di studio, Matera, 20-21 novembre 2007, Venosa: Osanna, 2009, p. 139-178.

RAGO, Margareth \& FUNARI, Pedro Paulo Abreu (eds.). Subjetividades antigas e modernas, São Paulo: Annablume, 2008.

RIDGWAY, David. The Italian Iron Age and Greece: from Hellenization to interaction. Festschrift in Honour of J. Richard Green, coll. Mediterranean archaeology. Supplement, Sydney: Dept. of Archeology, University of Sydney 2004, p. 7-14.

SAHLINS, Marshall. How 'natives' think: about captain Cook, for example, Chicago: University of Chicago Press, 1995.

SAID, Edward W. Culture and imperialism, New York: Knopf, 1993.

SAID, Edward W. Orientalism, Londres: Penguin, 2003.

Sibari V : relazione preliminare delle campagne di scavo 1973 (Parco del Cavallo ; Casa Bianca) e 1974 (Stombi ; Incrocio ; Parco del Cavallo ; Prolungamento Strada; Casa Bianca). coll. Notizie degli scavi di antichità. Atti della accademia nazionale dei Lincei, 42-43, Roma: Accademia nazionale dei Lincei, 1992.

SMALL, David B. Introduction, in SMALL, David B. (ed.). Methods in the Mediterranean: historical and archaeological views on texts and archaeology, Leiden: Brill, 1995, p. 1-22.

SMALL, David B. Monuments, laws, and analysis: combining archaeology and text in Ancient Athens, in SMALL, David B. (ed.). Methods in the Mediterranean: historical and archaeological views on texts and archaeology, Leiden: Brill, 1995, p. 143-174. 
SMALL, David B. The tyranny of the text: lost social strategies in current historical period archaeology in the classical Mediterranean, in FUNARI, Pedro Paulo Abreu, HALL, Martin \& JONES, Siân (eds.). Historical Archaeology: back from the edge, Londres: Routledge, 1999, p. 122-136.

SPIVAK, Gayatri Chakravorty. A critique of postcolonial reason : toward a history of the vanishing present, Cambridge (Mass): Harvard university press, 1999.

VAN DOMMELEN, Peter. Colonial constructs: colonialism and archaeology in the Mediterranean, World Archaeology, 28, n 3, 1997, p. 305-323.

VAN DOMMELEN, Peter. Colonial matters: material culture and postcolonial theory in colonial situations, in TILLEY, Christopher, KEANE, Webb, et alii (eds.). Handbook of Material Culture, Londres: Sage, 2006, p. 104-124.

VAN DOMMELEN, Peter. Postcolonial archaeologies between discourse and practice, World Archaeology, 43.1, 2011, p. 1-6.

VOSS, Barbara L. The Archaeology of ethnogenesis: race and sexuality in colonial San Francisco, 2a ed., Gainesville: University Press of Florida, 2015.

VOSS, Barbara L. \& CONLIN CASELLA, Eleanor (eds.). The Archaeology of colonialism: intimate encounters and sexual effects, New York: Cambridge University Press, 2012.

YNTEMA, Douwe Geert. Mental landscapes of colonization: the ancient written sources and the archaeology of early colonial-Greek southeastern Italy, Babesch, 75, 2000, p. 1-49. 